\title{
Neurological soft signs in obsessive-compulsive disorder: two empirical studies and meta-analysis
}

\author{
N. Jaafari ${ }^{1,2} \dagger$, L. Fernández de la Cruz ${ }^{1} \dagger$, M. Grau ${ }^{1,3}$, E. Knowles ${ }^{1}$, J. Radua ${ }^{1,4}$, S. Wooderson ${ }^{5}$, \\ C. Segalas ${ }^{6}$, P. Alonso ${ }^{6}$, M. L. Phillips ${ }^{7}$, J. M. Menchón ${ }^{6}$ and D. Mataix-Cols ${ }^{1 *}$ \\ ${ }^{1}$ King's College London, Institute of Psychiatry, London, UK \\ ${ }^{2}$ Centre Hospitalier Universitaire de Poitiers; CIC INSERM U 802 Poitiers; Faculté de Médecine et de Pharmacie, Université de Poitiers ; \\ Unité de Recherche Intersectorielle en Psychiatrie du Centre Hospitalier Henri Laborit, Poitiers, France \\ ${ }^{3}$ Department of Psychiatry, Hospital Clinic Universitari, Valencia, Spain \\ ${ }^{4}$ FIDMAG - CIBERSAM, Sant Boi de Llobregat, Spain \\ ${ }^{5}$ National Affective Disorder Unit, King's College London, London, UK \\ ${ }^{6}$ Department of Psychiatry, Bellvitge University Hospital, Barcelona, Spain \\ ${ }^{7}$ Department of Psychiatry, University of Pittsburgh School of Medicine, Pittsburgh, PA, USA
}

Background. Neurological soft signs (NSS) have been inconsistently reported in obsessive-compulsive disorder (OCD) but may make an impact on treatment response.

Method. The current study examined the presence of NSS in two independent European samples of OCD patients (combined 85 patients and 88 matched healthy controls) using a standardized instrument and conducted a metaanalysis of all published studies identified in the literature with the aim to provide a more definitive answer to the question of whether OCD patients are characterized by increased NSS.

Results. Both empirical studies found elevated NSS scores in patients compared with matched controls. The results of the meta-analysis, which included 15 studies (combined 498 patients and 520 controls) showed large effect sizes (Hedges' $g=1.27,95 \%$ confidence interval $0.80-1.75$ ), indicating that OCD patients have significantly higher rates of NSS than matched controls on both sides of the body and in multiple domains (motor coordination, sensory integration and primitive reflexes). The results were robust and remained largely unchanged in our reliability analyses, which controlled for possible outliers. Meta-regression was employed to examine the role of potential variables of interest including sociodemographic variables, symptom severity, medication effects and the use of different instruments, but none of these variables was clearly associated with NSS.

Conclusions. As a group, OCD patients are characterized by increased rates of NSS, compared with healthy controls. However, their origins and potential clinical importance remain to be clarified. Future directions for research are discussed.

Received 16 September 2011; Revised 13 July 2012; Accepted 20 July 2012; First published online 30 August 2012

Key words: Meta-analyses, neurological soft signs, obsessive-compulsive disorder.

\section{Introduction}

Conventionally defined as non-localizing abnormalities without diagnostic specificity, neurological soft signs (NSS) involve observable defects in sensory integration, motor coordination and primitive reflexes in patients without neurological disorder (Dazzan \& Murray, 2002). NSS have been described

\footnotetext{
* Address for correspondence: D. Mataix-Cols, Ph.D., PO Box 69, King's College London, Institute of Psychiatry, De Crespigny Park, London SE5 8AF, UK.

(Email: David.Mataix-Cols@kcl.ac.uk)

$\uparrow$ These authors contributed as first authors.
}

in several psychiatric disorders such as schizophrenia (Whitty et al. 2009; Chan et al. 2010b), bipolar disorder (Negash et al. 2004; Whitty et al. 2006), substance misuse (Keenan et al. 1997; Drvaux et al. 2010), antisocial personality disorder (Lindberg et al. 2004) and post-traumatic stress disorder (Gurvits et al. 1997).

In obsessive-compulsive disorder (OCD), a number of studies have explored NSS but the results have been relatively inconsistent. Some studies found a higher prevalence of NSS in OCD patients than in healthy controls (Conde López et al. 1990; Hollander et al. 1990; Bihari et al. 1991; Hymas et al. 1991; Nickoloff et al. 1991; Mataix-Cols et al. 2003; Guz \& Aygun, 2004; Sevincok et al. 2006; Salama et al. 2008), including 
impaired motor coordination (Hollander et al. 1990; Hymas et al. 1991; Bolton et al. 1998; Mataix-Cols et al. 2003), involuntary movements (Hollander et al. 1990; Bihari et al. 1991), sensory integration abnormalities (Bolton et al. 1998; Mataix-Cols et al. 2003; Sevincok et al. 2006) and presence of primitive reflexes (Bolton et al. 1998). However, other studies did not find any differences between OCD patients and controls in either total NSS scores (Stein et al. 1994; Jaafari et al. 2011), or in specific NSS subdomains such as motor coordination (Stein et al. 1994; Guz \& Aygun, 2004; Sevincok et al. 2006; Poyurovsky et al. 2007), sensory integration (Hollander et al. 1990; Stein et al. 1994; Poyurovsky et al. 2007), involuntary movements (Stein et al. 1994) or primitive reflexes (Mataix-Cols et al. 2003).

Detailed examination of these studies indicates that they are often characterized by relatively small sample sizes and various potential sources of heterogeneity. For example, in some studies patients were on medication (Bihari et al. 1991; Hymas et al. 1991; Nickoloff et al. 1991; Bolton et al. 1998; MataixCols et al. 2003; Guz \& Aygun, 2004; Poyurovsky et al. 2007; Jaafari et al. 2011) whereas other studies recruited unmedicated patients (Hollander et al. 1990; Stein et al. 1994; Sevincok et al. 2006). Another source of heterogeneity is the use of different standardized instruments to measure NSS, with some studies employing clinical examinations rather than standardized instruments (e.g. Hymas et al. 1991; Caramelli et al. 1996), thus making comparisons difficult.

Given the relative inconsistency of previous studies and the multiple sources of heterogeneity, the current study aimed to provide a more clear answer to the question of whether OCD patients are characterized by increased NSS compared with matched controls. This is clinically relevant in the light of emerging evidence that the presence of NSS in OCD may differentially predict treatment response to selective serotonin reuptake inhibitors (SSRIs) (Hollander et al. 2005; Mergl et al. 2005) but not cognitive behaviour therapy (Bolton et al. 2000). To this end, we conducted two empirical studies across two specialist European centres using the same standardized instrument. We next conducted exhaustive literature searches and performed a meta-analysis of all existing NSS studies in OCD. We predicted higher NSS scores in patients compared with controls in the empirical studies and robust effect sizes in the same direction in the meta-analysis. Finally, we employed meta-regression methods to examine the potential moderating role of symptom severity, medication use, age of onset, illness duration and other variables of interest.

\section{Method}

\section{Empirical studies}

Two parallel studies were conducted in the UK and Spain. The UK sample consisted of 74 individuals (35 with OCD and 39 controls) and the Spanish sample of 99 individuals (50 with OCD and 49 controls). In both samples, trained raters administered the Cambridge Neurological Inventory (CNI; Chen et al. 1995). A complete description of the participants, materials and methods can be found in the online Supplementary Material.

\section{Meta-analysis}

\section{Data sources and study selection}

A three-stage strategy was implemented to identify studies suitable for inclusion in the meta-analysis. First, we conducted a systematic search of the databases Medline, PsycINFO, PsychARTICLES, EMBASE, SCOPUS and Google Scholar using the search terms 'neurological soft signs', 'neurological signs', 'soft signs', 'neurological abnormalit*', 'motor coordination', 'sensory integration', 'complex motor sequencing', 'Luria task', or 'fist-edge-palm' with 'OCD' or 'obsessive compulsive disorder'. Second, we asked experts in the field to identify missing and unreported studies. Third, we conducted manual searches of reference lists of empirical studies and review articles. The inclusion criteria specified that the article must have included a control group and that the results must have been reported with sufficient detail for an effect size to be calculated.

\section{Data extraction and synthesis}

For each of the selected studies, two authors independently extracted the sample size of each group, the mean and standard deviation of the NSS scores, and the scores of the NSS subscales and the right and left NSS where available. Where possible, we also extracted information for a number of study characteristics. These variables were identified as potential sources of heterogeneity and were collected to be used as covariates in a meta-regression analysis.

Disagreement between the two authors concerning the extracted data was resolved via discussion until a consensus was reached. Any uncertainty regarding the extraction of data from a particular study was resolved via contact with the study authors.

Total NSS scores were not reported by Bolton et al. (1998) and Poyurovsky et al. (2007), so for these studies the NSS total score was calculated by summing the scores of the relevant subscales. 
Standard deviations of the total NSS scores were not reported in three papers (Hollander et al. 1990; Bolton et al. 1998; Poyurovsky et al. 2007) and had to be indirectly obtained. In one study (Hollander et al. 1990), these could be calculated from the histograms plotted in the paper. In another study (Bolton et al. 1998), standard deviations could be estimated from the subscores using a basic property of the variance, namely:

$\sigma_{A+B}=\sqrt{\sigma_{A}^{2}+\sigma_{B}^{2}+2 \sigma_{A} \sigma_{B} \rho_{A, B}}$,

where $\sigma_{A}$ and $\sigma_{A}$ are the standard deviations of two subscores and $\rho_{A, B}$ is the correlation between them - as reported in the original validation study of the CNI (Chen et al. 1995). Finally, a similar method was used for the data in Poyurovsky et al. (2007), though in this case the set of correlations reported in the original validation study of the Neurological Evaluation Scale (NES; Buchanan \& Heinrichs, 1989) led to a quadratic equation with two close roots for patients (1.47 and 2.24) and none for controls. In order to apply the same estimations for patients and controls we considered the discriminant of the equation to be null - i.e. equivalent to using the mean of the two estimated standard deviations in patients (1.86) and the optimal standard deviation in controls (1.54).

\section{Data analysis}

Meta-analyses were conducted using Stata, version 11 (StataCorp LP, USA). Hedges' $g$ was calculated for all measures; this method estimates the effect size as the standardized difference between group means, corrected for small sample size bias (Hunter \& Schmidt, 2000). A random-effects model was implemented, which estimates an error term for between-study variance based on the assumption that effect size magnitude varies between studies; this method is a conservative effect size estimate, provides more accurate estimates for confidence intervals (CIs) and substantially reduces the possibility of type I error (Hunter \& Schmidt, 2000; Field, 2005).

Next, a meta-influence analysis was conducted. The meta-influence analysis is a form of sensitivity analysis that allows examination of the possible influence of individual studies on the overall meta-analysis summary estimate. Using this method, an average effect size is calculated while leaving out one study at a time from the set of studies available for the metaanalysis.

In order to examine publication bias, we created a funnel plot and conducted Egger's test of publication bias (Egger et al. 1997) for each measure. If there is no evidence of publication bias, the individual studies should be symmetrically distributed within the funnel of the plot and Egger's test should be nonsignificant.

Heterogeneity was investigated using a metaregression analysis, which examines potential moderators of the effect size (Higgins \& Thompson, 2002). A lack of moderator effect suggests that the moderator in question does not affect the average effect size estimate. We computed the $\chi^{2}$ statistic $Q$ and the $I^{2}$ statistic in order to assess the homogeneity of effect sizes. Moderator analyses were conducted using random-effects meta-regression analyses with the study characteristics as covariates using the METAN and METAREG commands in Stata, version 11. Because of the increased risk of type I error when making multiple comparisons, we used a permutation tests approach (using 1000 Monte Carlo simulations) to calculate $p$ values (Higgins \& Thompson, 2004).

\section{Results}

\section{Empirical studies}

Compared with healthy controls, patients with OCD had elevated scores on most NSS subscales, particularly in the Spanish sample (Table 1). A detailed description of the results can be found in the Supplementary Material.

\section{Meta-analysis}

Our search strategy yielded 32 studies for potential inclusion. Of these, 15 met our inclusion criteria. Table 2 lists the included/excluded studies and reasons for exclusion.

The combined number of participants in these studies was 1018 (498 OCD and 520 control participants). One of the studies included in the metaanalysis (Stein et al. 1994) comprised 34 OCD subjects and 16 healthy controls, of which 16 OCD and eight control participants had been already reported in another of the included studies (Hollander et al. 1990). The analysis of the influence described in the methods controlled for this circumstance.

The combined sample characteristics were calculated taking into account the sample size and variances of each study. The combined sample consisted of individuals in their 30s (OCD: mean age $=33.5$ years, S.D. $=9.7$ years, range 28.5-36.9 years; controls: mean age $=33.6$ years, S.D. $=10.2$ years, range 29.638.2 years; 14 studies), with a similar gender distribution in both groups (OCD: mean percentage of females $=52 \%$; controls : mean percentage of females $=45 \%$; 14 studies), and similar years of education (OCD: mean $=12.8$ years, S.D. $=3.9$ years ; controls : mean $=13.7$ years, S.D. $=3.6$ years; seven studies). 
Table 1. Mann-Whitney $U$ tests scores showing group differences in NSS between OCD and control groups in the UK and Spanish samples

\begin{tabular}{|c|c|c|c|c|c|}
\hline \multirow[b]{2}{*}{ NSS variable } & \multicolumn{2}{|c|}{ Mean rank } & \multirow[b]{2}{*}{$U$} & \multirow[b]{2}{*}{$z$} & \multirow[b]{2}{*}{$p$} \\
\hline & OCD & Controls & & & \\
\hline \multicolumn{6}{|l|}{ UK sample } \\
\hline Subjects, $n$ & 35 & 39 & & & \\
\hline Primitive reflexes & 41.70 & 33.73 & 535.00 & -2.41 & 0.02 \\
\hline Motor coordination & 41.30 & 34.09 & 549.50 & -1.45 & 0.15 \\
\hline Sensory integration & 39.59 & 35.63 & 609.50 & -0.80 & 0.42 \\
\hline Total right NSS & 42.04 & 33.42 & 523.50 & -1.74 & 0.08 \\
\hline Total left NSS & 42.86 & 32.69 & 495.00 & -2.06 & 0.04 \\
\hline Total NSS & 42.50 & 33.01 & 507.50 & -1.90 & 0.06 \\
\hline \multicolumn{6}{|l|}{ Spanish sample } \\
\hline Subjects, $n$ & 50 & 49 & & & \\
\hline Primitive reflexes & 61.27 & 38.50 & 661.50 & -4.99 & 0.000 \\
\hline Motor coordination & 68.05 & 31.58 & 322.50 & -6.33 & 0.000 \\
\hline Sensory integration & 57.39 & 42.46 & 855.50 & -2.71 & 0.007 \\
\hline Total right NSS & 68.07 & 31.56 & 321.50 & -6.35 & 0.000 \\
\hline Total left NSS & 66.85 & 32.81 & 382.50 & -5.93 & 0.000 \\
\hline Total NSS & 68.38 & 31.24 & 306.00 & -6.44 & 0.000 \\
\hline
\end{tabular}

NSS, Neurological soft signs; OCD, obsessive-compulsive disorder.

For the OCD participants, the severity of the disorder, measured with the Yale-Brown Obsessive Compulsive Scale (YBOCS), was in the moderate range $($ mean $=23.7$, S.D. $=7.5$, range $17.9-28.5 ; 10$ studies). The duration of the illness ranged from 1 to 21 years $($ mean $=12.1$ years, s.D. $=10.3$ years; eight studies). The mean age of onset of the illness was 18.1 years (s.D. $=7.5$ years; five studies).

\section{Study characteristics}

Studies varied in terms of sample size (OCD: mean $=$ 33.2, S.D. $=13.4$, range $8-50$ participants; controls: mean $=34.7$, S.D. $=16.9$, range $12-67$ participants). Most of the studies were conducted in Europe $(66.7 \%), 26.7 \%$ in the USA, and one in North Africa $(6.7 \%)$

Most studies employed structured NSS batteries. Of the included studies, $26.7 \%$ used the CNI (Bolton et al. 1998; Mataix-Cols et al. 2003; current studies), 20\% used the NES (Nickoloff et al. 1991; Senincok et al. 2006; Poyurovsky et al. 2007) and 20\% used other validated batteries [two studies used the Physical and Neurological Examination for Soft Signs (PANESS): Guz \& Aygun, 2004; Salama et al. 2008; and one study used Krebs's scale: Jaafari et al. 2011]. Of the included studies, five studies $(35.7 \%)$ used a NSS clinical examination (Conde López et al. 1990; Hollander et al. 1990; Bihari et al. 1991; Hymas et al. 1991; Stein et al. 1994).
Only one of the included studies employed raters who were blind to the participants' patient or control status (Hollander et al. 1990).

\section{Publication bias}

Examination of the funnel plot (Fig. 1) showed that the majority of studies were grouped symmetrically around the mean effect size (with the exception of the outlier on the right-hand side of the plot; Bihari et al. 1991), suggesting no evidence of publication bias. Egger's test was significant $(7.2$, S.E. $=2.8, p<0.05)$ but removal of the outlier study (Bihari et al. 1991) from the analysis resulted in a non-significant Egger's test $(3.3$, s.E. $=2.5 ; p>0.05)$ in line with the original appraisal of the funnel plot.

\section{Effect sizes}

The effect size calculated for the total NSS was large at $1.27[Q=158.5$, degrees of freedom $(\mathrm{df})=14, p<0.001$; 15 studies] (Fig. 2). A total of five studies reported data for total left and total right NSS. The effect sizes calculated for both left and right NSS were large at 0.72 $(Q=21.2, \mathrm{df}=4, p<0.001)$ and $0.68(Q=24.3, \mathrm{df}=4$, $p<0.001)$, respectively. Where available, scores for the NSS subscales were also obtained. Effect sizes for the different subscales were large with an effect size of $0.81(Q=6.9, \mathrm{df}=3, p<0.075, n=4)$ for primitive reflexes, $0.67(Q=33.2, \mathrm{df}=7, p<0.000, n=8)$ for sensory integration and $0.61(Q=104.3, \mathrm{df}=8$, 
Table 2. Summary of the included and excluded studies in the meta-analysis

\begin{tabular}{|c|c|c|c|c|}
\hline \multirow[b]{2}{*}{ Study } & \multicolumn{2}{|c|}{ Subjects, $n$} & \multirow[b]{2}{*}{ NSS assessment } & \multirow[b]{2}{*}{$\begin{array}{l}\text { Included/excluded } \\
\text { (reasons for exclusion) }\end{array}$} \\
\hline & OCD & $\begin{array}{l}\text { Healthy } \\
\text { controls }\end{array}$ & & \\
\hline Hollander et al. (1990) & 41 & 20 & Clinical examination & Included \\
\hline Conde López et al. (1990) & 20 & 20 & Clinical examination & Included \\
\hline Bihari et al. (1991) & 39 & 43 & Clinical examination & Included \\
\hline Nickoloff et al. (1991) & 8 & 12 & NES & Included \\
\hline Hymas et al. (1991) & $17^{\mathrm{a}}$ & 16 & Clinical examination & Included \\
\hline Stein et al. (1994) & 34 & 16 & Clinical examination & Included \\
\hline Bolton et al. (1998) & 50 & 67 & CNI & Included \\
\hline Mataix-Cols et al. (2003) & 30 & 30 & $\mathrm{CNI}$ & Included \\
\hline Guz \& Aygun (2004) & 30 & 30 & PANESS & Included \\
\hline Sevincok et al. (2006) & 25 & 23 & NES & Included \\
\hline Poyurovsky et al. (2007) & 20 & 51 & NES & Included \\
\hline Salama et al. (2008) & 50 & 50 & PANESS & Included \\
\hline Jaafari et al. (2011) & 49 & 54 & Krebs's scale & Included \\
\hline Current study 1 (UK) & 35 & 39 & $\mathrm{CNI}$ & Included \\
\hline Current study 2 (Spain) & 50 & 49 & CNI & Included \\
\hline Bolton et al. (2000) & 35 & N.A. & $\mathrm{CNI}$ & Excluded (no control group) \\
\hline Behar et al. (1984) & 7 & N.A. & Clinical examination & $\begin{array}{l}\text { Excluded (no control group and missing } \\
\text { key information) }\end{array}$ \\
\hline Caramelli et al. (1996) & 15 & 15 & Clinical examination & Excluded (missing key information) \\
\hline Denckla (1989) & 54 & N.A. & PANESS & $\begin{array}{l}\text { Excluded (no control group and missing } \\
\text { key information) }\end{array}$ \\
\hline Hollander et al. (1991) & 12 & 12 & Clinical examination & $\begin{array}{l}\text { Excluded (mixed group and missing } \\
\text { key information) }\end{array}$ \\
\hline Hollander et al. (2005) & 117 & N.A. & Clinical examination & Excluded (no control group) \\
\hline Sawle et al. (1991) & 6 & N.A. & Clinical examination & Excluded (NSS data reported elsewhere) \\
\hline Schilder (1938) & 7 & N.A. & Clinical examination & $\begin{array}{l}\text { Excluded (no control group \& missing } \\
\text { key information) }\end{array}$ \\
\hline Singla et al. (2009) & 30 & N.A. & NES & $\begin{array}{l}\text { Excluded (no control group and missing } \\
\text { key information) }\end{array}$ \\
\hline Stein et al. (1993) & 16 & 8 & Clinical examination & Excluded (NSS data reported elsewhere) \\
\hline Stein et al. (1997) & 13 & 12 & Clinical examination & Excluded (missing key information) \\
\hline Thienemann et al. (1995) & 21 & N.A. & Clinical examination & Excluded (no control group) \\
\hline Thomsen \& Jensen (1991) & 61 & 117 & Clinical examination & Excluded (missing key information) \\
\hline Towey et al. (1993) & 17 & 16 & Clinical examination & Excluded (missing key information) \\
\hline Towey et al. (1994) & 18 & 15 & Clinical examination & Excluded (missing key information) \\
\hline Tumkaya et al. (2012) & 30 & N.A. & NES & Excluded (no healthy control group) \\
\hline
\end{tabular}

OCD, Obsessive-compulsive disorder; NSS, neurological soft signs; NES, Neurological Evaluation Scale; CNI, Cambridge Neurological Inventory; PANESS, Physical and Neurological Examination for Soft Signs; N.A., not applicable.

${ }^{a}$ All patients in this study were specifically selected and displayed obsessional slowness.

$p<0.000, n=10)$ for motor coordination (see Supplementary Material, Figs S1-S5).

The sensitivity analysis that excluded each study at the time (influence analysis) indicated that none of the individual studies exerted particular influence on the average effect size (Table 3).

\section{Meta-regression analysis}

For total NSS scores, variation in effect size that was attributable to heterogeneity was high $\left(I^{2}=91.2 \%\right)$.
Meta-regression analyses were therefore conducted in order to investigate possible sources of heterogeneity and their influence on NSS total findings. The potential moderator variables examined in reference to the study characteristics were the year of publication, the instrument used for the assessment of the NSS, and the continent where the study was done. The putative patient characteristic moderators also studied were gender, handedness, years of education, intelligence quotient (IQ), age of illness onset, duration of the 


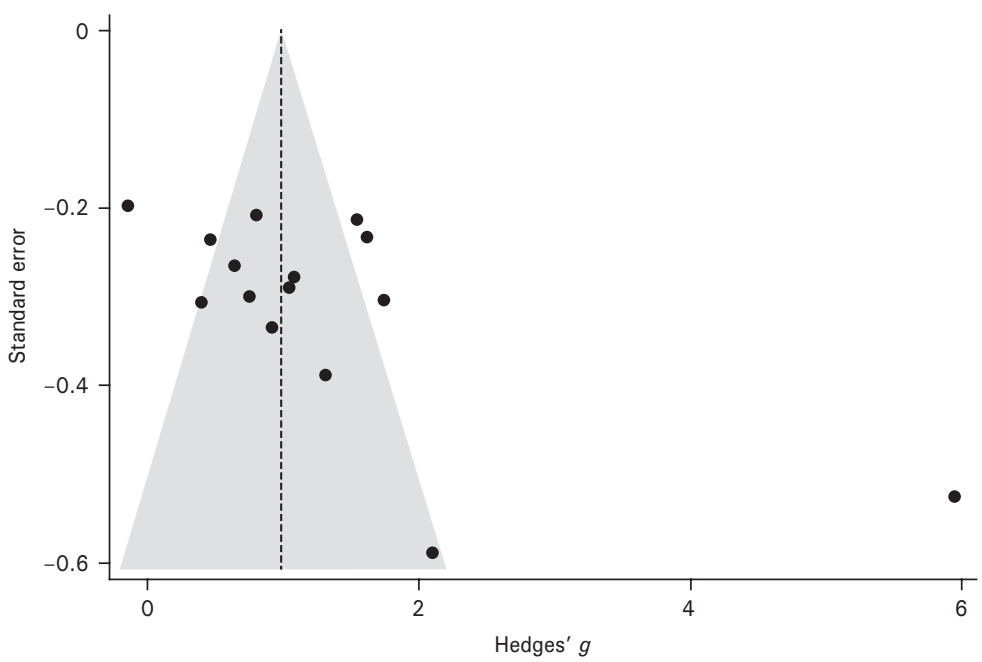

Fig. 1. Funnel plot for the meta-analysis of total neurological soft signs score in obsessive-compulsive disorder patients versus healthy controls.

Study

Hollander et al. (1990)

Conde López et al. (1990)

Bihari et al. (1991)

Hymas et al. (1991)

Nickoloff et al. (1991)

Stein et al. (1994)

Bolton et al. (1998)

Mataix-Cols et al. (2003)

Guz \& Aygun (2004)

Sevincock et al. (2006)

Poyurovsky et al (2007)

Salama et al. (2008)

Jaafari et al. (2011)

Current study 1 (UK)

Current study 2 (Spain)

Total

$\chi_{14}^{2}=158.47$
Hedges' $g$ Weight

(random)
$95 \% \mathrm{Cl}$

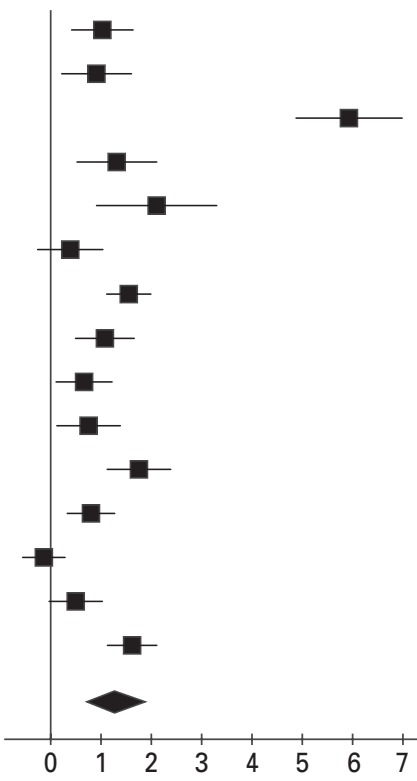

$1.05(0.48-1.62) \quad 6.80$

$0.92(0.27-1.58) \quad 6.59$

$5.95(4.92-6.98) \quad 5.57$

$1.31(0.55-2.07) \quad 6.32$

$2.11(0.95-3.26) \quad 5.22$

$0.40(-0.20-1.00) \quad 6.73$

$1.55(1.13-1.96) \quad 7.12$

$1.08(0.54-1.62) \quad 6.86$

$0.65(0.13-1.17) \quad 6.91$

$0.75(0.17-1.34) \quad 6.76$

$1.74(1.15-2.34) \quad 6.74$

$0.80(0.40-1.21) \quad 7.14$

$-0.14(-0.53-0.25) \quad 7.17$

$0.47(0.00-0.93) \quad 7.03$

$1.62(1.17-2.08) \quad 7.04$

$1.27(0.80-1.75) \quad 100.00$

Fig. 2. Forest plot for total neurological soft signs score in obsessive-compulsive disorder patients versus healthy controls. CI, Confidence interval.

illness, medication use, OCD severity and presence of depressive symptoms.

Of the potential moderator variables examined, none reached statistical significance, including YBOCS score (Supplementary Material, Fig. S6). However, there was a trend towards significance for year of publication $(\beta=-0.60, p=0.15)$, such that the more recently a study was published the smaller its effect size but this was probably due to the effect of an outlier (Bihari et al. 1991) (Supplementary Material, Fig. S7). A trend-level relationship was also found for the NSS instrument used $(\beta=-0.42, p=0.13)$, such that studies that used a clinical examination yielded larger effect sizes followed by studies that used the NES battery, the CNI, and finally the PANESS (Supplementary Material, Fig. S8). However, this may be explained by the unusually large effect sizes reported by Bihari et al. (1991).

\section{Discussion}

This study examined the presence of NSS in two large European samples of OCD patients and conducted a 
Table 3. Results of the influence analysis in which the effect sizes are re-estimated omitting one study at the time

\begin{tabular}{|c|c|c|}
\hline Study omitted & $\begin{array}{l}\text { Effect size } \\
\text { estimate }\end{array}$ & $(95 \% \mathrm{CI})$ \\
\hline Hollander et al. (1990) & 1.30 & $(0.79-1.81)$ \\
\hline Conde López et al. (1990) & 1.30 & $(0.80-1.81)$ \\
\hline Bihari et al. (1991) & 0.98 & $(0.65-1.31)$ \\
\hline Hymas et al. (1991) & 1.27 & $(0.77-1.78)$ \\
\hline Nickoloff et al. (1991) & 1.23 & $(0.74-1.72)$ \\
\hline Stein et al. (1994) & 1.34 & $(0.84-1.84)$ \\
\hline Bolton et al. (1998) & 1.26 & $(0.75-1.77)$ \\
\hline Mataix-Cols et al. (2003) & 1.29 & $(0.78-1.81)$ \\
\hline Guz \& Aygun (2004) & 1.33 & $(0.82-1.84)$ \\
\hline Sevincok et al. (2006) & 1.32 & $(0.81-1.82)$ \\
\hline Poyurovsky et al. (2007) & 1.24 & $(0.74-1.74)$ \\
\hline Salama et al. (2008) & 1.32 & $(0.79-1.84)$ \\
\hline Jaafari et al. (2011) & 1.37 & $(0.91-1.84)$ \\
\hline Current study 1 (UK) & 1.34 & $(0.83-1.85)$ \\
\hline Current study 2 (Spain) & 1.25 & $(0.75-1.76)$ \\
\hline Combined & 1.27 & $(0.80-1.75)$ \\
\hline
\end{tabular}

CI, Confidence interval.

meta-analysis of all published studies identified in the literature with the aim to provide a more definitive answer to the question of whether OCD patients are characterized by increased NSS compared with matched controls. Both empirical studies found elevated NSS scores in patients compared with matched controls, although the Spanish sample yielded more clearly significant results. The results of the metaanalysis revealed large effect sizes in the expected direction. The results were robust and remained largely unchanged in our reliability analyses, which controlled for possible outliers.

The results confirm the initial findings of Hollander et al. (1990) and Conde López et al. (1990) and clarify some of the previous inconsistencies in the literature, such as the specific types of NSS that are implicated in OCD; our results confirm that patients have higher rates of NSS in the domains of motor coordination, sensory integration and primitive reflexes. The results further indicate that both sides of the body are implicated in OCD, although only five studies provided laterality information. Some previous studies had suggested a slight predominance of left-sided NSS in OCD (e.g. Hollander et al. 1990; Mataix-Cols et al. 2003) but both the empirical studies and the metaanalysis showed robust bilateral differences between patients and controls.

The results echo other complementary lines of research, such as kinematic analyses of handwriting and facial movements (Mavrogiorgou et al. 2001; Mergl et al. 2003) and performance on oculo-motor tasks (Jaafari et al. 2011). Together, these findings help reconcile previous inconsistencies in the literature, which may have been caused by small heterogeneous samples, and suggest subtle central nervous system alterations in OCD which are broadly consistent with current neurobiological models of the disorder (e.g. Saxena \& Rauch, 2000). However, alternative explanations are also possible (see below).

Of the two empirical studies, the Spanish sample yielded larger effect sizes and tighter CIs. These discrepancies may be due to differences in the patient or control characteristics, the smaller sample size or increased variability in the UK sample, or a combination of these factors. Also, the scoring of the CNI was slightly different in the two studies, with the Spanish study adding an additional 'subthreshold' option. However, the influence analyses clearly indicated that the overall results of the meta-analysis were largely unaffected by the contribution of any of the individual studies.

Whereas the Spanish study found a significant association between NSS scores and OCD severity (YBOCS scores), the UK study did not (see Supplementary Material). The previous literature is also mixed in this regard. While some studies found significant associations between NSS and YBOCS scores (e.g. Hollander et al. 1990; Bolton et al. 2000; Salama et al. 2008), others did not (e.g. Stein et al. 1994, 1997; Sevincok et al. 2006; Jaafari et al. 2011; Karadag et al. 2011). Similarly, our meta-regression analysis also failed to confirm a significant association with symptom severity. This negative finding may be due to the relatively small variability in YBOCS scores across studies. Alternatively, NSS may be unrelated to fluctuations in OCD symptom severity and, rather, represent a trait marker of the disorder. As reviewed in the introduction, NSS are observed in multiple mental disorders, not just OCD. Thus, NSS may be nonspecific markers of psychopathology in general. This raises the question of whether NSS may also be present in first-degree relatives of OCD patients and thus represent useful endophenotypes for OCD research, as has been suggested in schizophrenia (Chan et al. $2010 a$ ). Examination of NSS in unaffected first-degree relatives of OCD patients would be a worthwhile avenue for future research.

Interestingly, in both empirical studies, NSS scores significantly correlated with the severity of depressive symptoms (see Supplementary Material). Unfortunately, we did not have sufficient data to conduct a meta-regression analysis to confirm these findings. If confirmed, one parsimonious interpretation may be that low mood interferes with appropriate performance on the NSS tasks, rather than represent a marker of central nervous system dysfunction. 
Alternatively, NSS may be associated with an increased risk and severity of psychiatric symptoms in general, including depression, although the current design does not allow us to examine the direction of such findings. In any case, comparison of OCD patients with and without co-morbid depression is needed in order to further clarify this issue (in the current study, only seven patients in each of the empirical samples met diagnostic criteria for major depression and thus meaningful comparisons were not possible).

Years of education were significantly (negatively) correlated with NSS in both empirical studies (see Supplementary Material) but this variable could not be examined in the meta-regression due to the lack of sufficient data. The design of the current study does not permit causal interpretations but it is possible that individuals with subtle neurological deficits represent a particularly impaired subgroup of OCD patients, characterized by learning difficulties and cognitive impairment. For example, Hertzig (1981) found that children with soft signs were significantly more likely to have received special education and to have been referred for psychiatric consultation than were neurologically normal children. This is also consistent with a body of evidence that links increased NSS with poorer neuropsychological performance in OCD (Hollander et al. 1990; Bolton et al. 2000; Mataix-Cols et al. 2003) and other psychiatric disorders (e.g. Flashman et al. 1996; Arango et al. 1999). Nevertheless, the alternative interpretation (i.e. that poorer educational attainment is responsible for the impaired NSS performance in OCD) cannot be fully ruled out at this stage.

None of the other potentially moderating variables we examined (i.e. gender, handedness, IQ, age of illness onset, duration of the illness, or medication use) was significantly associated with NSS in OCD. Regarding the lack of medication effects, the results are consistent with several studies that found no influence of medication on NSS in OCD (Bihari et al. 1991; Hymas et al. 1991; Thienemann \& Koran, 1995; Caramelli et al. 1996; Bolton et al. 1998; Guz \& Aygun, 2004; Poyurovsky et al. 2007; Salama et al. 2008; Jaafari et al. 2011; Karadag et al. 2011), or in other disorders such as schizophrenia (Heinrichs \& Buchanan, 1988; Chan et al. 2010b) or bipolar disorder (Cherian \& Kuruvilla, 1989; Noroozian et al. 2009). Perhaps antipsychotics may have more clearly moderating effects on NSS, but only four studies included in this metaanalysis contained a small proportion of patients who were prescribed low doses of antipsychotics as an augmentation strategy (Hymas et al. 1991; Poyurovsky et al. 2007; Jaafari et al. 2011; current study - Spanish sample). In any case, a recent meta-analysis of NSS studies in schizophrenia concluded: 'the majority of studies demonstrated no significant results suggesting that [antipsychotic] treatment might not be a significant moderator' (Chan et al. 2010b; p. 1097). Thus, based on the available evidence, it appears unlikely that medication played a substantial role in the current results.

\section{Directions for future research}

As seen above, several important questions remain but the current findings point towards specific areas that require further research.

First, it is currently unclear whether NSS precede the development of OCD or are a consequence of the disorder. Prospective longitudinal studies are needed to fully address this question, although there are some indications in the literature that NSS may precede and represent a risk factor for OCD as well as other mental disorders. In a rare prospective study, Grisham et al. (2011) examined childhood risk factors for an adult OCD diagnosis in a New Zealand birth cohort. They reported that poor motor skills in childhood (ages 3-9 years) specifically predicted the presence of OCD symptoms involving fears of harm and checking rituals. These results are consistent with a previous study by the same group, which demonstrated that deficits in visuospatial, visuoconstructive and visuomotor skills in childhood were specifically associated with an adult diagnosis of OCD (Grisham et al. 2009). Other researchers have also reported that NSS in childhood are predictive of a variety of psychiatric disorders later in life (Shaffer et al. 1983; Hollander et al. 1991; Fish et al. 1992; Pine et al. 1993; Jones et al. 1994). Therefore, NSS may represent neurodevelopmental risk factors for a variety of psychiatric conditions, including OCD. However, it cannot be entirely ruled out that at least some of the reported findings are secondary to OCD symptoms, as there is some evidence that NSS and other motor abnormalities can improve with successful treatment. For example, a study by Mergl et al. (2004) found that drawing speed was impaired in OCD compared with healthy controls but normalized after 10 -week treatment with sertraline and behaviour therapy. Another potentially useful way to address the state versus trait question in OCD may be the comparison of symptomatic versus fully remitted patients or, as mentioned earlier, the study of unaffected first-degree relatives of OCD patients.

Another area of interest is whether the NSS have any prognostic value in OCD and therefore have a potential use in clinical settings. It has been suggested that NSS may be useful predictors of treatment response to SSRIs in OCD. For example, Hollander et al. (2005) found that left-sided visuospatial soft signs 
were significantly increased in non-responders compared with responders to SSRIs. Meanwhile, Mergl et al. (2005) found that non-responders to a combination of sertraline and behaviour therapy exhibited more motor soft signs at baseline, compared with treatment responders. However, Thienemann \& Koran (1995) failed to find a link between NSS and response to SSRIs. Moreover, from the limited evidence, it does not seem that NSS are predictive of clinical response to behaviour therapy for OCD (Bolton et al. 2000). Taken together, the findings of this limited body of research raise the intriguing possibility that NSS assessment may be useful to guide treatment strategies in OCD but clearly more research is needed to establish their potential clinical use.

\section{Limitations}

One of the main limitations of the two empirical studies and the vast majority of studies included in the meta-analysis (except Hollander et al. 1990) was that examiners, though trained in most cases, were not blind to diagnosis, thus potentially resulting in the over-rating of NSS in the patients. This was partially overcome in the UK study, where $40 \%$ of the participants were double scored by a second examiner and the inter-rater reliability was found to be generally excellent (see Supplementary Material). It is also noteworthy that the effect size obtained in the metaanalysis is very similar to that obtained in the original Hollander et al. (1990) study, which did employ a blind methodology. Another important limitation is the cross-sectional nature of the research, which precludes firm conclusions regarding the nature and origin of NSS in OCD; prospective longitudinal studies are needed. Different studies employed different instruments to assess NSS but our meta-regression and subgroup analyses showed that the results were robust and independent of the instruments used. A similar meta-analysis in schizophrenia also found that the use of different instruments had no noticeable effect on the results (Chan et al. 2010b). Several important variables such as co-morbidity with tic disorders or depression, specific OCD symptom dimensions, obsessional slowness, or degree of insight were not systematically included in the original studies and therefore could not be investigated in the meta-analysis. It may be that NSS are particularly prominent in certain groups of patients and thus examination of these variables remains an objective for future research.

\section{Conclusions}

As a group, patients with OCD have elevated rates of subtle neurological signs, which rather than being specific to OCD, may represent markers of psychopathology in general. However, the origins and potential clinical importance of NSS in OCD remain to be clarified.

\section{Supplementary material}

For supplementary material accompanying this paper visit http://dx.doi.org/10.1017/S0033291712002012.

\section{Acknowledgements}

N.J. was supported by the Région Poitou-Charentes, ' 1 'École Doctorale Biosanté du PRES Limousin-PoitouCharentes', and the Centre hospitalier universitaire (CHU) de Poitiers. L.F.C. was supported by a fellowship from the Alicia Koplowitz Foundation.

\section{Declaration of Interest}

None.

\section{References}

Arango C, Bartko JJ, Gold JM, Buchanan RW (1999). Prediction of neuropsychological performance by neurological signs in schizophrenia. American Journal of Psychiatry 141, 1349-1357.

Behar D, Rapoport JL, Berg CJ, Denckla MB, Mann L, Cox C, Fedio P, Zahn T, Wolfman MG (1984). Computerized tomography and neuropsychological test measures in adolescents with obsessive-compulsive disorder. American Journal of Psychiatry 141, 363-369.

Bihari K, Pato MT, Hill JL, Murphy DL (1991). Neurologic soft signs in obsessive-compulsive disorder. Archives of General Psychiatry 48, 278-279.

Bolton D, Gibb W, Lees A, Raven P, Gray JA, Chen E, Shafran R (1998). Neurological soft signs in obsessive compulsive disorder: standardised assessment and comparison with schizophrenia. Behavioral Neurology 11, 197-204.

Bolton D, Raven P, Madronal-Luque R, Marks IM (2000). Neurological and neuropsychological signs in obsessive compulsive disorder: interaction with behavioural treatment. Behavioral Research and Therapy 38, 695-708.

Buchanan RW, Heinrichs DW (1989). The Neurological Evaluation Scale (NES) : a structured instrument for the assessment of neurological signs in schizophrenia. Psychiatry Research 27, 335-350.

Caramelli P, de Lima MA, Stip E, Bacheschi LA (1996). Neurological examination in obsessive-compulsive disorder. Sao Paulo Medical Journal 114, 1255-1258.

Chan RC, Xu T, Heinrichs RW, Yu Y, Gong QY (2010a). Neurological soft signs in non-psychotic first-degree relatives of patients with schizophrenia: a systematic review and meta-analysis. Neuroscience and Biobehavioral Reviews 34, 889-896. 
Chan RC, Xu T, Heinrichs RW, Yu Y, Wang Y (2010b). Neurological soft signs in schizophrenia: a meta-analysis. Schizophrenia Bulletin 36, 1089-1104.

Chen EY, Shapleske J, Luque R, McKenna PJ, Hodges JR, Calloway SP, Hymas NF, Dening TR, Berrios GE (1995). The Cambridge Neurological Inventory: a clinical instrument for assessment of soft neurological signs in psychiatric patients. Psychiatry Research 56, 183-204.

Cherian A, Kuruvilla K (1989). Prevalence of neurological 'soft signs' in affective disorder and correlation with response to treatment. Indian Journal of Psychiatry 31, 224-229.

Conde López V, de la Gándara Martin JJ, Blanco Lozano ML, Cerezo Rodriguez P, Martinez Roig M, de Dios Francos A (1990). Minor neurological signs in obsessive-compulsive disorders [article in Spanish]. Actas Luso-Espanolas de Neurologia, Psiquiatria y Ciencias Afines 18, 143-164.

Dazzan P, Murray RM (2002). Neurological soft signs in first-episode psychosis: a systematic review. British Journal of Psychiatry Supplement 43, p s50-s57.

Denckla MB (1989). Neurological examination. In Obsessive-Compulsive Disorder in Children and Adolescents (ed. J. L. Rapoport), pp. 107-115. American Psychiatric Press Inc. : New York.

Dervaux A, Bourdel MC, Laqueille X, Krebs MO (2010). Neurological soft signs in non-psychotic patients with cannabis dependence. Addiction Biology. Published online: 4 November 2010. doi:10.1111/j.1369-1600.2010.00261.x.

Egger M, Smith GD, Schneider M, Minder C (1997). Bias in meta-analysis detected by a simple graphical test. British Medical Journal 315, 629-634.

Field AP (2005). Meta-analysis. In A Handbook of Research Methods for Clinical and Health Psychology (ed. J. Miles and P. Gilbert), pp. 295-308. Oxford University Press: New York.

Fish B, Marcus J, Hans SL, Auerbach JG, Perdue S (1992). Infants at risk for schizophrenia: sequelae of a genetic neurointegrative defect. A review and replication analysis of pandysmaturation in the Jerusalem Infant Development Study. Archives of General Psychiatry 49, 221-235.

Flashman LA, Flaum M, Gupta S, Andreasen NC (1996). Soft signs and neuropsychological performance in schizophrenia. American Journal of Psychiatry 153, 526-532.

Grisham JR, Anderson TM, Poulton R, Moffitt TE, Andrews G (2009). Childhood neuropsychological deficits associated with adult obsessive-compulsive disorder. British Journal of Psychiatry 195, 138-141.

Grisham JR, Fullana MA, Mataix-Cols D, Moffitt TE, Caspi A, Poulton R (2011). Risk factors prospectively associated with adult obsessive-compulsive symptom dimensions and obsessive-compulsive disorder. Psychological Medicine 41, 2495-2506.

Gurvits TV, Gilbertson MW, Lasko NB, Orr SP, Pitman RK (1997). Neurological status of combat veterans and adult survivors of sexual abuse PTSD. Annals of the New York Academy of Sciences 821, 468-471.

Guz H, Aygun D (2004). Neurological soft signs in obsessive-compulsive disorder. Neurology India 52, 72-75.
Heinrichs DW, Buchanan RW (1988). Significance and meaning of neurological signs in schizophrenia. American Journal of Psychiatry 145, 11-18.

Hertzig ME (1981). Neurological 'soft' signs in low-birthweight children. Developmental Medicine and Child Neurology 23, 778-791.

Higgins JPT, Thompson SG (2002). Quantifying heterogeneity in a meta-analysis. Statistics in Medicine 21, 1539-1558.

Higgins JPT, Thompson SG (2004). Controlling the risk of spurious findings from meta-regression. Statistics in Medicine 23, 1663-1682.

Hollander E, DeCaria CM, Aronowitz B, Klein DF, Liebowitz MR, Shaffer D (1991). A pilot follow-up study of childhood soft signs and the development of adult psychopathology. Journal of Neuropsychiatry and Clinical Neurosciences 3, 186-189.

Hollander E, Kaplan A, Schmeidler J, Yang H, Li D, Koran LM, Barbato LM (2005). Neurological soft signs as predictors of treatment response to selective serotonin reuptake inhibitors in obsessive-compulsive disorder. Journal of Neuropsychiatry and Clinical Neurosciences 17, 472-477.

Hollander E, Schiffman E, Cohen B, Rivera-Stein MA, Rosen W, Gorman JM, Fyer AJ, Papp L, Liebowitz MR (1990). Signs of central nervous system dysfunction in obsessive-compulsive disorder. Archives of General Psychiatry 47, 27-32.

Hunter JE, Schmidt FL (2000). Fixed effects vs. random effects meta-analysis models: implications for cumulative research knowledge. International Journal of Selection and Assessment 8, 275-292.

Hymas N, Lees A, Bolton D, Epps K, Head D (1991). The neurology of obsessional slowness. Brain 114, 2203-2233.

Jaafari N, Baup N, Bourdel MC, Olié JP, Rotge JY, Wassouf I, Sharov I, Millet B, Krebs MO (2011). Neurological soft signs in OCD patients with early age at onset compared with patients with schizophrenia and healthy subjects. Journal of Neuropsychiatry and Clinical Neurosciences 23, 409-416.

Jones P, Rodgers B, Murray R, Marmot M (1994). Child development risk factors for adult schizophrenia in the British 1946 birth cohort. Lancet 344, 1398-1402.

Karadag F, Tumkaya S, Kırtaş D, Efe M, Alacam H, Oguzhanoglu NK (2011). Neurological soft signs in obsessive compulsive disorder with good and poor insight. Progress in Neuropsychopharmacology and Biological Psychiatry 35, 1074-1079.

Keenan E, O'Donnell C, Sinanan K, O'Callaghan E (1997). Severity of alcohol dependence and its relationship to neurological soft signs, neuropsychological impairment and family history. Acta Psychiatrica Scandinavica 95, 272-276.

Lindberg N, Tani P, Stenberg JH, Appelberg B, Porkka-Heiskanen T, Virkkunen M (2004). Neurological soft signs in homicidal men with antisocial personality disorder. European Psychiatry 19, 433-437.

Mataix-Cols D, Alonso P, Hernández R, Deckersbach T, Savage CR, Manuel Menchón J, Vallejo J (2003). Relation of neurological soft signs to nonverbal 
memory performance in obsessive-compulsive disorder. Journal of Clinical and Experimental Neuropsychology 25, 842-851.

Mavrogiorgou P, Mergl R, Tigges P, El Husseini J, Schröter A, Juckel G, Zaudig M, Hegerl U (2001). Kinematic analysis of handwriting movements in patients with obsessive-compulsive disorder. Journal of Neurology, Neurosurgery, and Psychiatry 70, 605-612.

Mergl R, Mavrogiorgou P, Juckel G, Zaudig M, Hegerl U (2004). Effects of sertraline on kinematic aspects of hand movements in patients with obsessive-compulsive disorder. Psychopharmacology 171, 179-185.

Mergl R, Mavrogiorgou P, Juckel G, Zaudig M, Hegerl U (2005). Can a subgroup of OCD patients with motor abnormalities and poor therapeutic response be identified? Psychopharmacology 179, 826-837.

Mergl R, Vogel M, Mavrogiorgou P, Göbel C, Zaudig M, Hegerl U, Juckel G (2003). Kinematical analysis of emotionally induced facial expressions in patients with obsessive-compulsive disorder. Psychological Medicine 33, 1453-1462.

Negash A, Kebede D, Alem A, Melaku Z, Deyessa N, Shibire T, Fekadu A, Fekadu D, Jacobsson L, Kullgren G (2004). Neurological soft signs in bipolar I disorder patients. Journal of Affective Disorders 80, 221-230.

Nickoloff SE, Radant AD, Reichler R, Hommer DW (1991). Smooth pursuit and saccadic eye movements and neurological soft signs in obsessive-compulsive disorder. Psychiatry Research 38, 173-185.

Noroozian M, Amini H, Faridhosseini F, Irandoost P, Saghaie T (2009). Neurological soft signs: a further step in the diagnosis of bipolar-I disorder. Iranian Journal of Psychiatry 4, 7-12.

Pine D, Shaffer D, Schonfeld IS (1993). Persistent emotional disorder in children with neurological soft signs. Journal of the American Academy of Child and Adolescent Psychiatry 32, 1229-1236.

Poyurovsky M, Faragian S, Pashinian A, Levi A, Viosburd A, Stryjer R, Weizman R, Fuchs C, Weizman A (2007). Neurological soft signs in schizophrenia patients with obsessive-compulsive disorder. Journal of Neuropsychiatry and Clinical Neurosciences 19, 145-150.

Salama HM, Saad Allah HM, Mohamed NA (2008). Study of the neurological soft signs in a sample of obsessivecompulsive patients and its correlation with the severity of obsessive-compulsive symptoms and the degree of insight. Alexandria Bulletin 44, 9-12.

Sawle GV, Hymas NF, Lees J, Frackowiak RDJ (1991). Obsessional slowness: functional studies with positron emission tomography. Brain 114, 2191-2202.

Saxena S, Rauch SL (2000). Functional neuroimaging and the neuroanatomy of obsessive-compulsive disorder. Psychiatric Clinics of North America 23, 563-586.

Schilder P (1938). The organic background of obsessions and compulsions. American Journal of Psychiatry 94, 1397-1416.

Sevincok L, Akoglu A, Arslantas H (2006). Schizo-obsessive and obsessive-compulsive disorder: comparison of clinical characteristics and neurological soft signs. Psychiatry Research 145, 241-248.

Shaffer D, O'Connor PA, Shafer SQ, Prupis S (1983).

Neurological 'soft signs' : their origins and significance for behavior. In Developmental Neuropsychiatry (ed. M. Rutter), pp. 144-163. Guilford: New York.

Singla N, Singh P, Gargi PD (2009). A comparative study of soft neurological signs in patients of schizophrenia and obsessive-compulsive disorder. Oral presentation at the 2nd European Conference on Schizophrenia Research: From Research to Practice, 21-23 September 2009, Berlin, Germany. European Archives of Psychiatry and Clinical Neuroscience 259 (Suppl. 1), S55.

Stein DJ, Coetzer R, Lee M, Davids B, Bouwer C (1997). Magnetic resonance brain imaging in women with obsessive-compulsive disorder and trichotillomania. Psychiatry Research 74, 177-182.

Stein DJ, Hollander E, Chan S, DeCaria CM, Hilal S, Liebowitz MR, Klein DF (1993). Computed tomography and neurological soft signs in obsessive-compulsive disorder. Psychiatry Research 50, 143-150.

Stein DJ, Hollander E, Simeon D, Cohen L, Islam MN, Aronowitz B (1994). Neurological soft signs in female trichotillomania patients, obsessive-compulsive disorder patients, and healthy control subjects. Journal of Neuropsychiatry and Clinical Neurosciences 6, 184-187.

Thienemann M, Koran LM (1995). Do soft signs predict treatment outcome in obsessive-compulsive disorder? Journal of Neuropsychiatry and Clinical Neurosciences 7, 218-222.

Thomsen PH, Jensen J (1991). Latent class analysis of organic aspects of obsessive-compulsive disorder in children and adolescents. Acta Psychiatrica Scandinavica 84, 391-395.

Towey J, Bruder G, Tenke C, Leite P, DeCaria C, Friedman D, Hollander E (1993). Event-related potential and clinical correlates of neurodysfunction in obsessivecompulsive disorder. Psychiatry Research 49, 167-181.

Towey JP, Tenke CE, Bruder GE, Leite P, Friedman D, Liebowitz M, Hollander E (1994). Brain event-related potential correlates of overfocused attention in obsessivecompulsive disorder. Psychophysiology 31, 535-543.

Tumkaya S, Karadag F, Orguzhanoglu NK (2012). Neurological soft signs in schizophrenia and obsessive-compulsive disorder spectrum. European Psychiatry: the Journal of the Association of European Psychiatrists 27, 192-199.

Whitty P, Clarke M, McTigue O, Browne S, Gervin M, Kamali M, Lane A, Kinsella A, Waddington J, Larkin C, O'Callaghan E (2006). Diagnostic specificity and predictors of neurological soft signs in schizophrenia, bipolar disorder and other psychoses over the first 4 years of illness. Schizophrenia Research 86, 110-117.

Whitty PF, Owoeye O, Waddington JL (2009). Neurological signs and involuntary movements in schizophrenia: intrinsic to and informative on systems pathobiology. Schizophrenia Bulletin 35, 415-424. 